\title{
FEM analysis of temperature distribution of flat type mold for CFRTP thermoforming with direct resistance heating
}

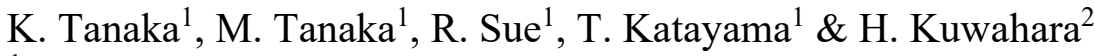 \\ ${ }^{I}$ Department of Biomedical Engineering, Doshisha University, Japan \\ ${ }^{2}$ Material Processing Technologies (MPT), Japan
}

\begin{abstract}
Carbon Fiber Reinforced Plastics (CFRP) are expected to be used for lightweight component parts of automobiles. Considering recyclability and productivity, Carbon Fiber Reinforced Thermoplastics (CFRTP) have been getting attention. However, the cost of CFRTP is so high that the use of CFRTP is rarely applied in the automobile industries. In order to reduce the cost of CFRTP, a novel production process with short cycle time needs to be developed. Direct resistance heating of the mold for CFRTP thermoforming is the technology in which high speed heating can be obtained by using high frequency voltage. However, uniformity of temperature distribution is one of the important issues to be solved. In this study, in order to understand the proximity effect, effect of electrode shapes and the frequency of applied voltage on the temperature distribution of a flat type mold, FEM analysis of the electromagnetic field and heat transfer was conducted and analysed results were compared with the experimental results. When alternative current is applied to the facing surfaces of molds in different directions in FEM analysis, the current concentrates to the facing surface due to the proximity effect. The analysed temperature of the mold surface corresponds to the experimental results. When higher frequency is applied to the mold, higher concentration of current is obtained. When the electrodes are mounted to be close to the mold surface, the mold surface can be efficiently heated.

Keywords: Carbon Fiber Reinforced Thermoplastics (CFRTP), direct resistance heating, proximity effect, frequency, electrode shapes, FEM analysis.
\end{abstract}




\section{Introduction}

In the automobile industry, it is necessary to reduce the weight of cars from the environmental aspect [1]. Carbon Fiber Reinforced Plastics (CFRP), which have high specific strength and specific rigidity, are expected to be used for lightweight component parts [2]. Considering recyclability and productivity, Carbon Fiber Reinforced Thermoplastics (CFRTP), which use thermoplastic for the matrix, have been getting attention. However, the cost of CFRTP is so high that the use of CFRTP is rarely applied in the automobile industries [3].

In order to reduce the cost of CFRTP, new molding methods and new materials are required. A novel production process with short cycle time is needed to be developed to reduce the production cycle time and to obtain the cost reduction. For high-speed heating methods of the mold, there are electromagnetic induction heating and direct resistance heating [4-6]. When the heating target is larger, electromagnetic induction heating needs large coils. Therefore, it makes the equipment more complicated and the production cost becomes higher. Direct resistance heating of the mold for CFRTP thermoforming is the technology in which high speed heating can be obtained by using high frequency voltage. This method can be run with simple equipment and low consumed power and reduce the equipment cost. However, uniformity of temperature distribution is one of the important issues to be solved.

In a previous study, pipe mold is successfully heated by direct resistance heating, and the effects of the frequency and electrode shapes are discussed [7, 8]. However, in the matched die with upper and lower molds, these effects and the proximity effect, in which current concentrates to the facing surface when alternative current is applied to the facing surfaces of the molds in different directions, are not clear. In this study, in order to reveal the effects of electrode shapes and the frequency of the applied voltage on the temperature distribution of a flat type mold, FEM analysis of the electromagnetic field and heat transfer was conducted and the analysed results were compared with the experimental results.

\section{FEM analysis and experimental methods}

\subsection{Governing equation}

COMSOL Multiphysics (COMSOL AB) was used for FEM analysis. Electric and Induction Currents (AC/DC Module) and General Heat Transfer (Heat Transfer Module) were used. Considering frequency properties, magnetic permeability as $\mu^{\prime}$ and conductivity as $\sigma^{\prime}$ are expressed in the following equations (1) and (2).

$$
\begin{gathered}
\mu^{\prime}=i \omega \sigma-\omega^{2} \varepsilon_{0} \varepsilon_{r} \\
\sigma^{\prime}=\sigma+i \omega \varepsilon_{0} \varepsilon_{r}
\end{gathered}
$$

$\omega$ is angular frequency, $\sigma$ is conductivity of the conductor, $\varepsilon_{0}$ is dielectric constant of vacuum, and $\varepsilon_{r}$ is dielectric constant of the conductor. The governing equation of $\mathrm{AC} / \mathrm{DC}$ Module is expressed in the following equations (3) and (4).

$$
-\nabla \cdot\left(\mu^{\prime} A+\sigma^{\prime} \nabla V-J\right)=0
$$




$$
\mu^{\prime} A+\nabla \times\left(\mu_{0}^{-1} \mu_{r}^{-1} \nabla \times A\right)+\sigma^{\prime} \nabla V=J
$$

$\mu_{0}$ is dielectric constant of vacuum, $\mu_{r}$ is dielectric constant of the conductor, $J$ is current, $A$ is magnetic field, and $\mathrm{V}$ is electric potential. The governing equation of heat transfer module is expressed in the following equation (5).

$$
\rho C_{P} \frac{\partial T}{\partial t}-\nabla(k \nabla T)=Q
$$

$\rho$ is density, $C_{p}$ is specific heat, $T$ is temperature, $t$ is time, $k$ is heat conduction coefficient, and $Q$ is heat source. Heat source is calculated from the current density distribution. The coupled analysis is performed based on these expressions.

\subsection{Analysed model and conditions}

Analysed model is a pair of flat type mold of rectangular parallelepiped of $40 \mathrm{~mm}$ in width, $80 \mathrm{~mm}$ in length, and $20 \mathrm{~mm}$ in height. The mold is made of SUS430 and S50C. Schematic drawing of the proximity effect is shown in Figure 1. Thanks to the proximity effect, mold surfaces can be efficiently heated by joule heat. Copper was used for the material of the electrode. The molds were surrounded by a layer of air to consider heat dissipation to the air. The relative magnetic permeability, electric conductivity and thermal conductivity of the materials used in analysis are shown in Table 1. For boundary condition, continuity was applied in analysis of electromagnetic field, and natural convection was applied in the analysis of heat transfer. Electrodes were attached to both ends of the mold. Electrodes of the copper plate were grounded on one end and potential was applied to the other end. The initial temperature was set at $20^{\circ} \mathrm{C}$ and heating time was set to 60 seconds. In order to reveal the effect of the electrode shapes, two types of analysed models for electrode shown in Figure 2 were used. While the electrodes of Type-A are connected to close to the facing surface of the mold, these of Type$\mathrm{B}$ are far. Input power was set to $1.7 \mathrm{~kW}$ and the frequency was set to $185 \mathrm{kHz}$. In order to reveal the effect of the frequency on the temperature distribution, the four levels of frequency, $10 \mathrm{kHz}, 50 \mathrm{kHz}, 100 \mathrm{kHz}$ and $185 \mathrm{kHz}$, were applied to the

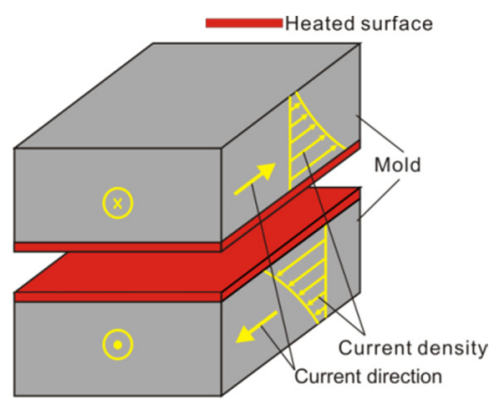

Figure 1: Schematic drawing of proximity effect. 
384 High Performance and Optimum Design of Structures and Materials II

Type-A model. The temperature distribution on Line A shown in Figure 3 was measured for each analysis.

Table 1: $\quad$ Properties of material.

\begin{tabular}{c|c|c|c|c}
\hline \hline & Air & Copper & S50C & SUS430 \\
\hline Relative magnetic permeability & 1 & 1 & 170.0 & 122.4 \\
\hline Electric conductivity $[\mathrm{S} / \mathrm{m}]$ & 0 & $5.998 \times 10^{7}$ & $3.378 \times 10^{6}$ & $1.666 \times 10^{6}$ \\
\hline $\begin{array}{c}\text { Thermal conductivity } \\
{[\mathrm{W} /(\mathrm{m} \cdot \mathrm{K})]}\end{array}$ & 0.257 & 400 & 26.0 & 25.6 \\
\hline
\end{tabular}

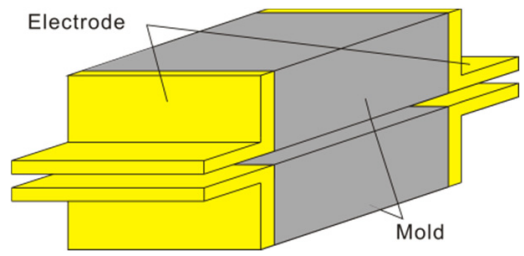

(a) Type-A.

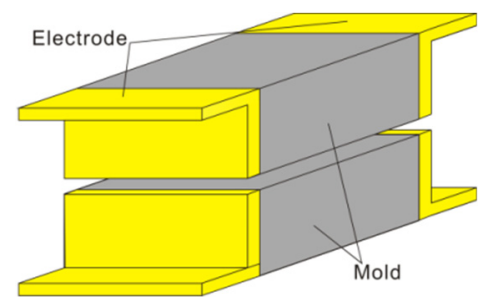

(b) Type-B.

Figure 2: $\quad$ Analysed models for electrodes.

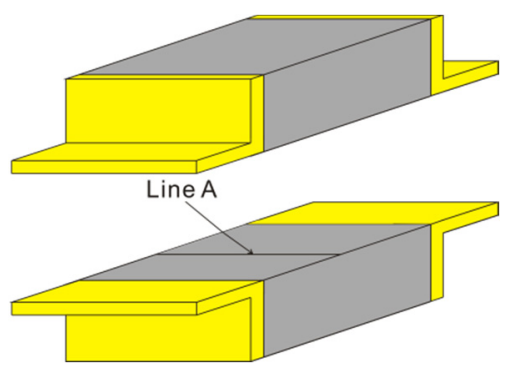

Figure 3: Temperature measured line. 


\subsection{Experimental method}

Schematic drawing of experimental system is shown in Figure 4. Two blocks of carbon steel of type S50C with the dimension of $40 \mathrm{~mm}$ in width, $80 \mathrm{~mm}$ in length, and $20 \mathrm{~mm}$ in height were used as the mold material, and they were connected by $1 \mathrm{~mm}$ of copper plate. A Teflon sheet of $2 \mathrm{~mm}$ in thickness was inserted between the molds as electrical insulation. K-type thermocouples were attached to four points and the temperatures were recorded with a graphic recorder (OMRON, Portable Multi Logeer). Output power of $1 \mathrm{~kW}$ and current frequency of $185 \mathrm{kHz}$ were supplied by the vacuum-tube oscillator (NETUREN, V-5) until the temperature of the surfaces reached $150^{\circ} \mathrm{C}$.

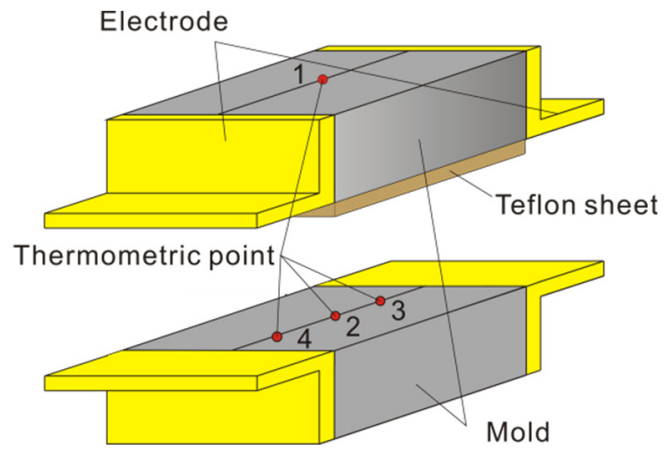

Figure 4: Schematic drawing of experimental system.

\section{Results and discussion}

\subsection{Effect of the electrode shapes}

Temperature distribution and magnetic field distribution of Type-A and Type-B for SUS430 are shown in Figures 5 and 6 respectively. In Type-A heated the facing surface of the mold are heated more efficiently than that in Type-B. The magnetic field generated by current flowing through the electrodes was concentrated on the facing surface of the mold. To obtain high speed heating of the molds, the arrangement methods of an electrode in Type-A is better than that in Type-B. 


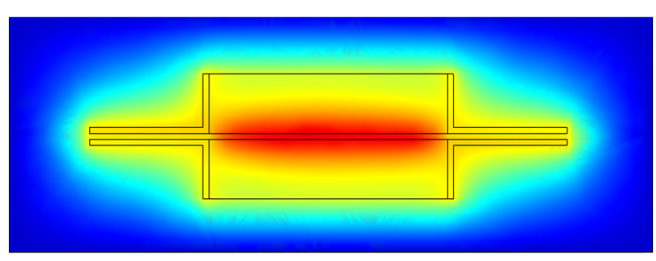
$280\left[{ }^{\circ} \mathrm{C}\right]$

(a) Type-A.

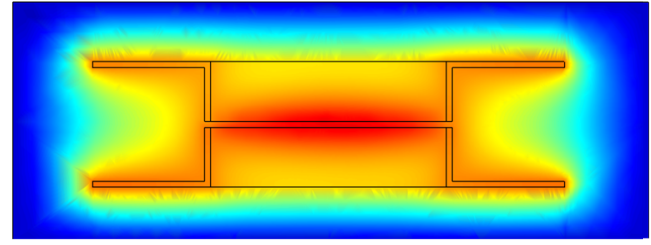

(b) Type-B.

Figure 5: Temperature distribution.

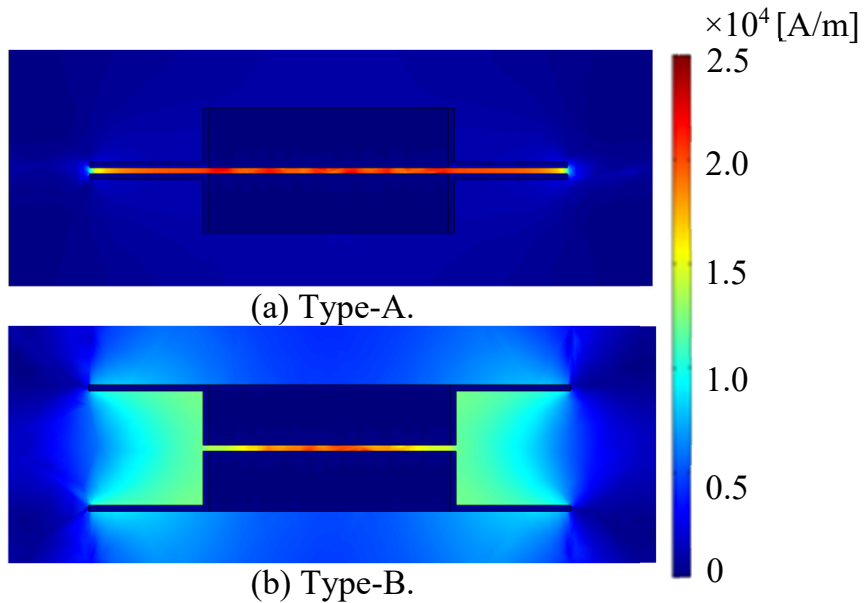

Figure 6: Magnetic field distribution.

\subsection{Effect of the frequency on the temperature distribution of the molds}

Temperature history of each of the frequency on Line A and distribution of current density at $10 \mathrm{kHz}$ and $185 \mathrm{kHz}$ for SUS430 are shown in Figures 7 and 8 respectively. As the frequency becomes smaller, the maximum temperature becomes lower. Concentration of the current density of frequency of $185 \mathrm{kHz}$ is 
larger than that of $10 \mathrm{kHz}$. High frequency can provide higher the proximity effect and high speed heating.

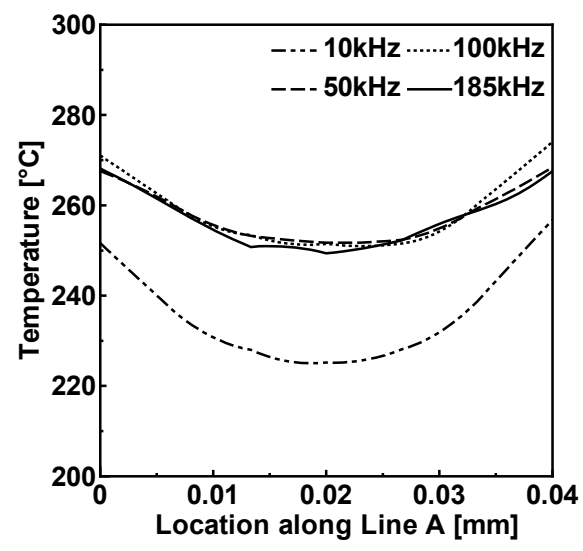

Figure 7: Temperature history.

$$
\times 10^{7}[\mathrm{~A} / \mathrm{m}]
$$
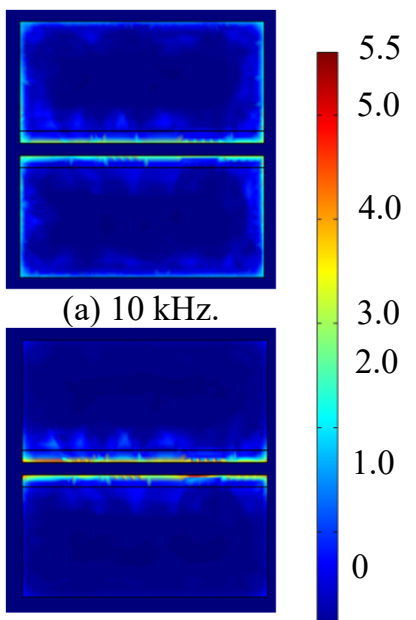

(b) $185 \mathrm{kHz}$.

Figure 8: Current density distribution.

\subsection{Comparison between analysed results and experimental results}

Temperature history of the analysed results and experimental results for S50C are shown in Figure 9. There is temperature difference above $38^{\circ} \mathrm{C}$ between Point1, which is on the outer surface of the mold, and Points 2 or 3 or 4 . While heating time to reach $150^{\circ} \mathrm{C}$ of Points 2 and 4 was 40 seconds in the analysis, it was 100 seconds in the experiment. In the experiment, output power by the vacuum-tube 
oscillator was set to $1 \mathrm{~kW}$, but the actual input power to the molds was less due to the loss of the reflection waves. Although the heating time of analysis does not correspond to the experimental results, the shape of the temperature history has good agreement with the experimental results.

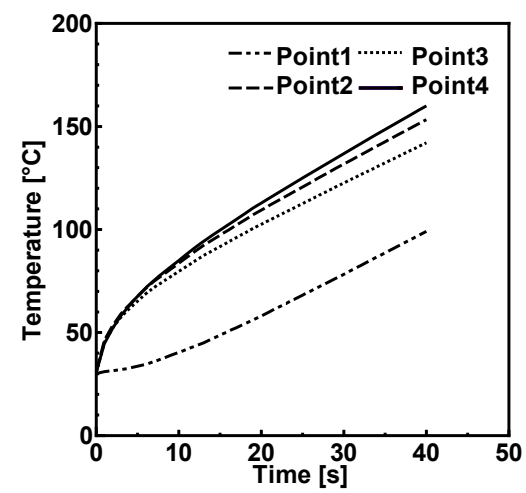

(a) Analysed results.

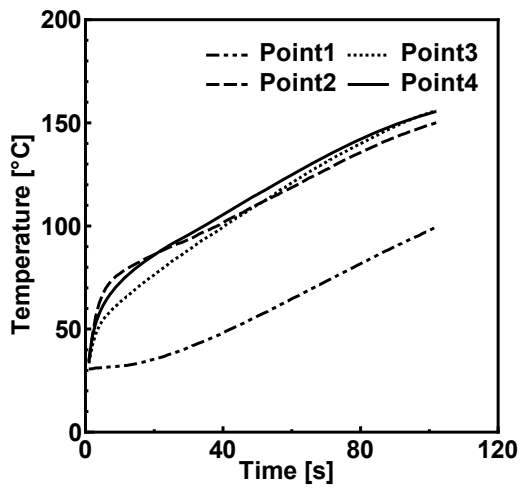

(b) Experimental results.

Figure 9: Temperature history.

\section{Conclusion}

To reveal the effect of electrode shapes and the frequency of the applied voltage on the temperature distribution of a flat type mold, FEM analysis of the electromagnetic field and heat transfer was conducted and analysed results were compared with the experiment results. The investigation yields the following conclusions.

1. The proximity effect is confirmed by both the FEM analysis and the experiment. The shape of the temperature history has a good agreement with the experimental results. As the frequency of applied voltage becomes 
higher, temperature of the proximate surface of the mold tends to increase thanks to the proximity effect.

2. As the electrodes are mounted to be close to the facing surface of the mold, the facing surface of the mold can be efficiently heated.

\section{References}

[1] Feraboli, P., Masini, A., Taraborrelli, L. \& Pivetti, A., Integrated development of CFRP structures for a topless high performance vehicle, Composite Structures, Vol. 78, pp. 495-506, 2007.

[2] Daisho, Y., Perspectives on Future Motor Vehicle Technologies Associated with Environment and Energy, IATSS Review, Vol. 33, No. 3, pp. 51-56, 2008.

[3] Wadahara, E. \& Kitano, A., Automotive lightweight structural elements of carbon fiber reinforced plastics, Sen'i Gakkaishi, Vol. 64, No. 9, pp. 295-301, 2008.

[4] Tanaka, K., Kohashi, N., Kinoshita, Y., Katayama, T. \& Uno, K., Compression molding process of carbon fiber reinforced thermoplastics using non-woven stitched multi-axial cloth by means of induction heating system, Journal of the Society of Materials Science, Vol. 58, No. 7, pp. 642-648, 2009.

[5] Perrier, D. \& Feigenblum, J., Rapid processing of tubular parts made of thermoplastic composites, JEC-Composites, pp. 68-70, 2008.

[6] Tanaka, K., Harada, R., Uemura, T., Katayama, T. \& Kuwahara, H., Rapid Pipe Moulding Process of Carbon Fiber Reinforced Thermoplastics by Highfrequency Direct Resistance Heating, WIT Transactions on the Built Environment, Vol. 112, pp. 133-139, 2010.

[7] Tanaka, K., Matsuura, Y., Harada, R., Katayama, T. \& Enoki, S., FEM analysis of temperature distribution of CFRTP pipe mold with direct resistance heating, WIT Transactions on the Built Environment, Vol. 137, pp. 265-272, 2014.

[8] Tanaka, K., Yamada, S., Matsuura, Y. \& Katayama, T., FEM analysis of temperature distribution of CFRTP curved pipe mold heated by highfrequency direct resistance heating, WIT Transactions on the Built Environment, Vol. 90, pp. 233-242, 2015. 